\title{
Universal properties of the $U(1)$ current at deconfined quantum critical points: comparison with predictions from gauge-gravity duality
}

\author{
Flavio S. Nogueire* \\ Institut für Theoretische Physik, Freie Universität Berlin, Arnimallee 14, D-14195 Berlin, Germany
}

(Dated: Received November 19, 2018)

\begin{abstract}
The deconfined quantum critical point of a two-dimensional $S U(N)$ antiferromagnet is governed by an Abelian Higgs model in $d=2+1$ spacetime dimensions featuring $N$ complex scalar fields. In this context, we derive for $2 \leq d \leq 4$ an exact formula for the central charge of the $U(1)$ current in terms of the gauge coupling at quantum criticality and compare it with the corresponding result obtained using gauge-gravity duality. There is a remarkable similarity precisely for $d=2+1$. In this case the amplitude of the current correlation function has the same form as predicted by the gauge-gravity duality. We also compare finite temperature results for the charge susceptibility in the large $N$ limit with the result predicted by the gauge-gravity duality. Our results suggest that condensed matter systems at quantum criticality may provide interesting quantitative tests of the gauge-gravity duality even in absence of supersymmetry.
\end{abstract}

PACS numbers: $64.70 . \mathrm{Tg}, 11.10 . \mathrm{Kk}, 11.25 . \mathrm{Tq}$

Correlation functions involving conserved physical quantities are in some special circumstances determined by simple dimensional analysis. This is the case for a theory at its quantum critical point. For relativistically invariant theories having a conserved $U(1)$ current $j_{\mu}(x)$, the current correlation function at criticality has a remarkable simple form, being given by [1]

$$
\left\langle j_{\mu}(x) j_{\nu}(0)\right\rangle=\frac{k}{S_{d}^{2} x^{2(d-1)}}\left(\delta_{\mu \nu}-\frac{2 x_{\mu} x_{\nu}}{x^{2}}\right),
$$

where $S_{d}=2 \pi^{d / 2} / \Gamma(d / 2)$ is the surface of a unit sphere in $d$ spacetime dimensions. The above result follows from the conformal invariance of the quantum critical point and the fact that (on-shell) conserved $U(1)$ currents do not have an anomalous dimension. Thus, the dimensionless number $k$ is the central charge of the $U(1)$ current in a $d$-dimensional conformal field theory $\left(\mathrm{CFT}_{d}\right)$.

Another dimensionless number, $k^{\prime}$, is defined at a quantum critical point and finite temperature via the charge susceptibility $\chi=\lim _{V \rightarrow \infty}\left\langle Q^{2}\right\rangle / V$, where $Q$ is the conserved charge associated to the $U(1)$ current, i.e., it is simply obtained by integrating $j_{0}(x)$ over $d-1$ spatial dimensions. Thus, dimensional analysis and universality implies

$$
\chi=k^{\prime} T^{d-2} .
$$

Recently, Kovtun and Ritz 2] have shown that for theories having a gravitational dual [3, 4], the following relation holds,

$$
r_{G}(d) \equiv \frac{k^{\prime}}{k}=\frac{1}{2 \pi^{d / 2}}\left(\frac{4 \pi}{d}\right)^{d-2} \frac{\Gamma^{3}(d / 2)}{\Gamma(d)},
$$

\footnotetext{
*Electronic address: nogueira@physik.fu-berlin.de
}

which for $d=2$ agrees with the exact result for a $\mathrm{CFT}_{2}$ [2], namely,

$$
\left.\frac{k^{\prime}}{k}\right|_{d=2}=\frac{1}{2 \pi},
$$

the latter being complementary to a well-known relation for the central charge of the energy-momentum tensor, $c$, and the universal amplitude of the free-energy [ [5, $[6]$.

It would be interesting to find examples where the result (3) holds. Note that this would not necessarily imply that a theory fulfilling (3) has a gravitational dual, but finding such an example would be very encouraging. In this paper we will show that condensed matter systems at quantum criticality may provide relatively simple quantitative tests of gravitational duality. We will derive results for $k$ and $k^{\prime}$ in effective theories of quantum antiferromagnets. These results will then be compared with those predicted by the gauge-gravity duality.

The quantum critical point of an $S U(N)$ quantum antiferromagnet is described by an Abelian Higgs model with $N$ complex bosons $[7,8]$, i.e.,

$$
\begin{aligned}
\mathcal{L} & =\frac{1}{4} F_{\mu \nu}^{2}+\sum_{a=1}^{N}\left|D_{\mu} z_{\alpha}\right|^{2} \\
& +r_{0} \sum_{a=1}^{N}\left|z_{\alpha}\right|^{2}+\frac{u_{0}}{2}\left(\sum_{a=1}^{N}\left|z_{\alpha}\right|^{2}\right)^{2},
\end{aligned}
$$

where $D_{\mu}=\partial_{\mu}+i e_{0} A_{\mu}$. Obviously, the conserved $U(1)$ current for the above Lagrangian is

$$
j_{\mu}=-i e_{0} \sum_{a=1}^{N}\left(z_{a}^{*} D_{\mu} z_{a}-z_{a} D_{\mu}^{*} z_{a}^{*}\right) .
$$

For a spin 1/2 antiferromagnet, the Lagrangian (5) follows from the representation of the spin orientation field as $\mathbf{n}(x)=z_{a}^{*}(x) \boldsymbol{\sigma}_{a b} z_{b}(x)$, where the fields $z_{a}$ represent the so called spinons and $\boldsymbol{\sigma}=\left(\sigma_{1}, \sigma_{2}, \sigma_{3}\right)$ is a vector 
having the Pauli matrices as components. The generalization to $S U(N)$ follows simply by replacing the Pauli matrices by the generators of $S U(N)$. From this representation a local gauge symmetry naturally emerges, since the spin orientation field $\mathbf{n}(x)$ remains invariant under the local phase transformation $z_{a}(x) \rightarrow e^{i \theta(x)} z_{a}(x)$. Perturbatively, this theory has a quantum critical point for large enough $N$ and $2<d<4$ [9, 10], in which case the spinons $z_{a}$ are deconfined in the sense defined precisely in Ref. [8]. A quantum critical point has been found non-perturbatively for all $N$ by means of the exact RG [11]. For $d=3$ spacetime dimensions this quantum critical point separates two distinct Mott insulating phases. In one of these phases the fields $z_{a}$ are condensed, leading in this way to antiferromagnetic (AF) order. This corresponds to a Higgs phase in particle physics terminology. The other phase is paramagnetic and corresponds in the lattice to an insulating pattern formed by a crystalline structure of singlet bonds, the so called valencebond solid (VBS) [12]. In particle physics language, this phase corresponds to a spinon confinement phase. The character of the AF-VBS phase transition for $S U(2)$ spins is at present controversial, with some numerical results in the lattice favoring a second-order phase transition (and therefore, the existence of a quantum critical point) [13], while other numerical results favor a weak first-order phase transition [14]. In this paper we will assume that a quantum critical point exists, which is definitly true if $N$ is large enough and $2<d<4[15]$. Precisely at the quantum critical point the theory is a $\mathrm{CFT}_{d}$.

Let us show that there exists an exact relation between the number $k$ in Eq. (1) and the dimensionless renormalized gauge coupling at the quantum critical point. The renormalized gauge coupling is uniquely determined by the vacuum polarization, as dictated by the Ward identities. The vacuum polarization, on the other hand, is related in momentum space to the current correlation function by

$$
\Pi(p)=-\frac{e_{0}^{2}}{(d-1) p^{2}}\langle j(p) \cdot j(-p)\rangle,
$$

where $e_{0}^{2}$ is the bare gauge coupling. Therefore, the renormalized gauge coupling $e^{2}(p)$ is given by the exact expression

$$
\frac{1}{e^{2}(p)}=\frac{1}{e_{0}^{2}}-\frac{1}{(d-1) p^{2}}\langle j(p) \cdot j(-p)\rangle .
$$

We are interested in the regime near or at the quantum critical point, so that $|p| \ll e_{0}^{2}$, which is the regime where Eq. (11) is valid. From Eq. (11) it is easy to obtain that

$$
\langle j(p) \cdot j(-p)\rangle=\frac{(d-2) \Gamma(1-d / 2) \Gamma^{2}(d / 2)}{(4 \pi)^{d / 2} \Gamma(d-1)} k|p|^{d-2} .
$$

The dimensionless gauge coupling is defined by $f(p)=$ $p^{d-4} e^{2}(p)$. Its critical value is given by the RG fixed point $f_{*}=\lim _{p \rightarrow 0} f(p)$. Therefore, we obtain from Eqs.
(8) and (9) that $k$ is exactly related to the fixed point $f_{*}$ via the formula:

$$
k=\frac{2^{d-1} \pi^{d / 2} \Gamma(d)}{\Gamma(2-d / 2) \Gamma^{2}(d / 2)} \frac{1}{f_{*}} .
$$

A similar relation holds in theories admitting a gravitational dual, with the difference that the central charge of the theory in the boundary is related to the gauge coupling of the theory in the bulk, which is also coupled to gravity in an AdS background. Indeed, an expression for the central charge $k$ in terms of the gauge coupling of the Einstein-Maxwell action in a $(d+1)$-dimensional anti-de Sitter $\left(\mathrm{AdS}_{d}\right)$ spacetime was derived by Freedman et al. [18]. The Einstein-Maxwell action is

$$
\begin{aligned}
S & =\frac{1}{16 \pi G} \int d^{d+1} x \sqrt{-g}[R+d(d-1) \Lambda] \\
& -\frac{1}{4 g_{d+1}^{2}} \int d^{d+1} x \sqrt{-g} F_{\mu \nu}^{2} .
\end{aligned}
$$

The cosmological constant in the above action sets the scale to define the dimensionless gauge coupling as $\hat{g}_{d+1}^{2}=\Lambda^{(d-3) / 2} g_{d+1}^{2}$. The $U(1)$ central charge $k$ of the $\mathrm{CFT}_{d}$ on the boundary is related to the gauge coupling of the theory in the bulk by [18]

$$
k=\frac{2 \pi^{d / 2}(d-2) \Gamma(d)}{\Gamma^{3}(d / 2)} \frac{1}{\hat{g}_{d+1}^{2}} .
$$

Note that, in contrast with Eq. (10), where $f_{*}$ is the coupling constant of the $\mathrm{CFT}_{d}$, in Eq. (12) $\hat{g}_{d+1}^{2}$ is the coupling constant of the theory with gravity. Remarkably, Eqs. (10) and (12) have exactly the same form for $d=3$ spacetime dimensions, where $g_{4}^{2}$ is dimensionless:

$$
k=\frac{32}{f_{*}}, \quad k=\frac{32}{g_{4}^{2}} .
$$

Despite the similarity between the two $k$ 's, we cannot safely claim that $f_{*}$ can be identified with $g_{4}^{2}$, since unfortunately the symmetries of the quantum antiferromagnet do not entirely match with the ones of the supergravity theory. Another important issue is that usually in applications involving the quark-gluon plasma [19] or quantum critical phenomena in condensed matter physics $[20,21,22]$ the current is associated to a global symmetry of the boundary theory. Here, in order to relate $k$ to the fixed point $f_{*}$, we have considered a current having a local gauge symmetry. In $S U(N)$ supersymmetric QCD, where the $\beta$ function is exactly known [23], many other interesting results for the central charges of gauge invariant currents are known [24].

As a concrete example, let us consider the large $N$ limit of the theory (5). In this case we have in $d$ spacetime dimensions,

$$
f_{*}=\frac{(4 \pi)^{d / 2}(d-2) \Gamma(d)}{4 \Gamma(2-d / 2) \Gamma^{2}(d / 2) N},
$$


which is just the fixed point of the RG one-loop $\beta$ function for a fixed dimensionality [25]. Thus, Eq. (10) yields

$$
k=\frac{2 N}{d-2},
$$

which is the same result as for a free scalar field theory featuring $N$ complex fields. Up to the factor $N$, this is also the same result as in the conformally invariant $O(n)$ model [26, 27]. For $d=3$ the fixed point (14) becomes $f_{*}=16 / N$. The four-dimensional gauge coupling constant of the gravitational theory, on the other hand, is given by $g_{4}^{2}=6 \pi /\left(\sqrt{2} N^{3 / 2}\right)$ [20]. Thus, we see that the gravitational theory yields a non-perturbative value of $k$, since in this case $k=16 \sqrt{2} N^{3 / 2} /(3 \pi)$ instead of giving a result $\sim \mathcal{O}(N)$.

If in addition we minimally couple the theory in (5) to $N_{f}$ Dirac fermions (with four-component spinors), corresponding to the algebraic charge liquid [10, 15, 16, 28], we obtain for large $N$ and $N_{f}$, with $N / N_{f}$ arbitrary, the result

$$
k=\frac{2 N+4(d-2) N_{f}}{d-2} .
$$

Thus, for the purely fermionic case we have $k=4 N_{f}$.

The above results can also be applied to the easy-plane system, by generalizing the global $U(1) \times U(1)$ symmetry to $O(N) \times O(N)$ with $N$ even [9]. Although the easy-plane antiferromagnet exhibits a first-order phase transition [29] (this is true for all $N$; see Ref. [9]), the central charge $k$ depends only on the fixed point $f_{*}$ and not on the other couplings of the theory. In this case a real value of $f_{*}$ can always be found for large enough $N$ in a minimal subtraction scheme. Indeed, the $\beta$ function of the gauge coupling $f$ is only a function of $f$, not depending on the other couplings of the theory. Resummation schemes actually indicate that $f_{*}$ may exist for all values of $N$ [30].

Let us give a clear example of a situation in gauge theories where a universal constant appears even in the absence of a second-order phase transition. A simple example is provided by the static particle-antiparticle potential calculated from the Wilson loop. Quite generally, the static interquark potential is given at large distances by $V(L)=\tau_{s} L-C / L+\mathcal{O}\left(1 / L^{2}\right)$, where $\tau_{s}$ is the string tension and $C$ is a universal constant. It turns out that the constant $C$ is always universal, even if no quantum critical points exist, like for example in the case of Polyakov's compact Maxwell electrodynamics [31], where no phase transition happens, i.e., the string tension is always nonzero. The reason for this can be stated in very simple terms [32]: the contribution $\propto T / L$ in the large $L$ expansion of $T V(L)$ is invariant under a scale transformation $T \rightarrow \lambda T, L \rightarrow \lambda L$. For a bosonic string model of quark confinement we have, for example, $C=(d-2) \pi / 24$ [33]. The constant $C$ is related to the current correlation function. Indeed, a simple calculation for an Abelian gauge theory yields

$$
C=\frac{S_{d}(d-2) f_{*}}{2^{d} \pi^{d-1}} .
$$

Although in the seld-dual easy-plane case, no quantum critical point arises, it is possible to find a zero for the $\beta$ function of the gauge coupling, so that $C$ will be a universal number. Similarly, other relations involving the current correlation function hold. For instance, the universal relations derived in Ref. [20] for the self-dual easyplane antiferromagnet seem to remain valid, although the system, at zero temperature, exhibits a first-order phase transition [9, 29].

An exact computation of $k^{\prime}$ in terms of $f_{*}$ is not as straightforward as in the case of the central charge $k$ of the $U(1)$ current. However, since at large $N$ the central charge $k$ has the same value as in a free theory with $N$ complex scalar fields [an $O(2 N)$-invariant theory], it is instructive to calculate $k^{\prime}$ for this free theory and compute the ratio $k^{\prime} / k$. The calculation of $\chi$ is more easily done by coupling $j_{0}(x)$ to a uniform source $h$ and computing $\chi=-\partial^{2} f /\left.\partial h^{2}\right|_{h=0}$, where $f$ is the free-energy density at the presence of the external source. This leads us to the result

$$
\chi=4 N T \sum_{n=-\infty}^{\infty} \int \frac{d^{d-1} p}{(2 \pi)^{d-1}} \frac{\omega_{n}^{2}}{\left(\omega_{n}^{2}+\mathbf{p}^{2}\right)^{2}},
$$

where $\omega_{n}=2 \pi n T$. By performing explicitly the Matsubara sum and the momentum integral, we obtain

$$
k^{\prime}=N \pi^{(d-5) / 2}(d-3) \Gamma\left(\frac{3-d}{2}\right) \zeta(3-d) .
$$

In particular, for $d=3$ we have $k^{\prime}=N / \pi$. Therefore, we obtain the ratio between the $k^{\prime}$ above and the $k$ given in Eq. (15) as

$$
r_{\text {free }}(d) \equiv \frac{k^{\prime}}{k}=\frac{\pi^{(d-5) / 2}(d-2)(d-3)}{2} \Gamma\left(\frac{3-d}{2}\right) \zeta(3-d) .
$$

This is the exact result for a free theory. Note that for $d=2$ it agrees with Eq. (4), as it should. Interestingly, the $d=2$ result is in this case the same as the $d=3$ one, i.e., $r_{\text {free }}(2)=r_{\text {free }}(3)=1 /(2 \pi)$. This actually reflects a more general property of $r_{\text {free }}(d)$, namely,

$$
r_{\text {free }}(d)=r_{\text {free }}(5-d) .
$$

The prediction for $k^{\prime}$ from the $\mathrm{AdS}_{d+1} / \mathrm{CFT}_{d}$ correspondence is 2]

$$
k^{\prime}=\frac{d-2}{\hat{g}_{d+1}^{2}}\left(\frac{4 \pi}{d}\right)^{d-2},
$$

which when divided by Eq. (12) gives the relation (3). Note that $r_{G}(3)=\pi / 24<r_{\text {free }}(3)$. In Fig. 1 we plot the ratios $r_{G}(d)$ and $r_{\text {free }}(d)$ in the range $2 \leq d \leq 4$. We see from the figure that $r_{G}(d) \leq r_{\text {free }}(d)$ in the interval $2 \leq d \leq 4$.

The value of the ratio $k^{\prime} / k$ for the $O(n)$ non-linear $\sigma$ model [27] at large $n$ and $d=3$ was given in Ref. 2] as 


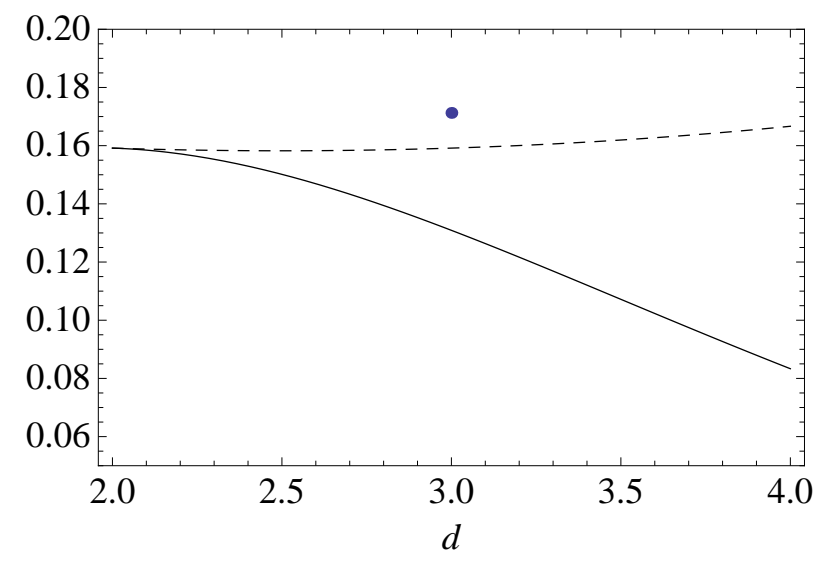

FIG. 1: Comparison between the ratios $r_{G}(d)$ and $r_{\text {free }}(d)$. The continuous line represents $r_{G}(d)$, while the dashed line corresponds to $r_{\text {free }}(d)$. The point represents the ratio $k^{\prime} / k$ for the $O(n)$ non-linear $\sigma$-model at large $n$ and $d=3$ [see Eq. (23)].

34

$$
r_{O(n)}=\frac{\sqrt{5}}{2 \pi} \ln \left(\frac{\sqrt{5}+1}{2}\right)
$$

which is about $7 \%$ larger than $r_{\text {free }}(3)$. Thus, for $d=3$ the free theory result is closer to the gravitational dual prediction than the corresponding large $n$ result for the $O(n)$ non-linear $\sigma$-model.

In Ref. 35] (see also Ref. [36]) it was conjectured that there is a duality between the critical interacting $O(n)$ model in $d=3$ and an even spin gauge theory defined in $\mathrm{AdS}_{4}$. It would be interesting to see such a gravitational dual construction working also in the case of the gauge theory formulation of quantum antiferromagnets.

In summary, we have seen that at zero temperature the quantum critical gauge coupling of an $S U(N)$ quantum antiferromagnet is related via an exact formula to the central charge $k$ of the conserved $U(1)$ current [Eq. (10)].
There is a corresponding formula for theories having a gravitational dual [18] [Eq. (12)]. However, it relates the gauge coupling of a $(d+1)$-dimensional gravitational theory to the central charge of the $U(1)$ current in a confomal theory living at the boundary. These expressions have the same form for $d=3$, where the gauge coupling of the gravitational theory is dimensionless. At finite temperature we have computed the amplitude $k^{\prime}$ of the charge susceptibility at quantum criticality in the large $N$ limit, which in this case is the same as the exact result for a model consisting of $N$ complex scalar fields. The ratio $k^{\prime} / k$ at large $N$ was compared with the prediction of the gauge-gravity duality. They both agree at $d=2$ with the exact result $k^{\prime} / k=1 /(2 \pi)$ for two-dimensional conformal field theories [2]. On the other hand, the derived large $N$ result for $k^{\prime} / k$ is found to differ at $d=3$ of about $18 \%$ from the gauge-gravity dual prediction. It would be interesting to see if better approximations can get closer to the gravitational duality result. One promising approach in this case is an expansion in $\epsilon=4-d$, where one would be able to give results for finite $N$. Another future project is to derive an exact formula for $k^{\prime}$ in terms of $f_{*}$, as we did with $k$. It would be interesting if such a formula for $d=3$ has a form similar to Eq. (22), in the same way as with $k$ in Eq. (13). However, it should be mentioned here that such a computation may lead instead to a breakdown of the universaly of the ratio $k^{\prime} / k$. For instance, in the context of the $\mathrm{AdS}_{d+1} / \mathrm{CFT}_{d}$ correspondence, Ritz and Ward [37] have recently shown that deviations from classical gravity in the bulk due to Weyl corrections in the Einstein-Maxwell action lead to a non-universal result for $k^{\prime} / k$.

\section{Acknowledgments}

The author would like to thank S. Sachdev for discussions and the Deutsche Forschungsgemeinschaft (DFG), grant No. KL 256/46-1, for the financial support.
[1] We have chosen here to follow Ref. [2] and include a factor $S_{d}^{2}$ in the definition of $k$ via the current correlation function.

[2] P. Kovtun and A. Ritz, Phys. Rev. D 78, 066009 (2008).

[3] J. M. Maldacena, Adv. Theor. Math. Phys. 2, 231 (1998); O. Aharony, S. S. Gubser, J. M. Maldacena, O. Oguri, and Y. Oz, Phys. Rep. 323, 183 (2000).

[4] For an accessible introduction to the subject, see I. R. Klebanov and J. M. Maldacena, Physics Today 62, 28 (2009).

[5] I. Affleck, Phys. Rev. Lett. 56, 746 (1986).

[6] H. W. J. Bloete, J. L. Cardy, and M. P. Nightingale, Phys. Rev. Lett. 56, 742 (1986).

[7] S. Sachdev, Nature Physics 4, 173 (2008).

[8] T. Senthil, A. Vishwanath, L. Balents, S. Sachdev, and
M. P. A. Fisher, Science, 303, 1490 (2004); T. Senthil, L. Balents, S. Sachdev, A. Vishwanath, and M. P. A. Fisher, Phys. Rev. B 70, 144407 (2004).

[9] F. S. Nogueira, S. Kragset, and A. Sudbø, Phys. Rev. B 76, 220403(R) (2007).

[10] F. S. Nogueira, Phys. Rev. B 77, 195101 (2008).

[11] B. Bergerhoff, D. Litim, S. Lola, and C. Wetterich, Int. J. Mod. Phys. A 11, 4273 (1996).

[12] N. Read and S. Sachdev, Phys. Rev. Lett. 62, 1694 (1989); Phys. Rev. B 42, 4568 (1990).

[13] A. W. Sandvik, Phys. Rev. Lett. 98, 227202 (2007); R. G. Melko and R. K. Kaul, Phys. Rev. Lett. 100, 017203 (2008).

[14] A. B. Kuklov, M. Matsumoto, N. V. Prokof'ev, B. V. Svistunov, and M. Troyer, Phys. Rev. Lett. 101, 050405 
(2008); F.-J. Jiang, M. Nyfeler, S. Chandrasekharan, and U.-J. Wiese, J. Stat. Mech. P02009 (2008).

[15] When Dirac fermions are included, it can be shown that a quantum critical point exists in $S U(2)$ antiferromagnets when the number of fermionic degrees of freedom $N_{f}>4$ [10]. Dirac fermions represent in this context the doping of the system near a spinon deconfinement phase transition. The resulting quantum liquid, the so called algebraic charge liquid [16], seems to describe well many of the features of high- $T_{c}$ superconductors in the underdoped regime. There are other situations where fermionic degrees of freedom are known to facilitate the emergence of a quantum critical point. For example, there is a supersymmetric model where deconfined quantum criticality is known to occur [17].

[16] R. K. Kaul, Y. B. Kim, S. Sachdev, and T. Senthil, Nature Physics 4, 28 (2008).

[17] S. Sachdev and X. Yin, arXiv:0808.0191.

[18] D. Z. Freedman, S. D. Mathur, A. Matusis, and L. Rastelli, Nucl. Phys. B 546, 96 (1999).

[19] D. T. Son and A. O. Starinets, Ann. Rev. Nucl. Part. Sci. 57, 95 (2007).

[20] C. P. Herzog, P. Kovtun, S. Sachdev, and D. T. Son, Phys. Rev. D 75, 085020 (2007).

[21] S. A. Hartnoll, P. K. Kovtun, M. Mueller, and S. Sachdev, Phys. Rev. B 76,144502 (2007).

[22] S. A. Hartnoll, "Lectures on holographic methods for condensed matter physics", arXiv:0903.3246.

[23] V Novikov, M. A. Shifman, A. I. Vainshtein, and V. Za- kharov, Nucl. Phys. B 229, 381 (1983).

[24] D. Anselmi, D. Z. Freedman, M. T. Grisaru, and A. A. Johansen, Nucl. Phys. B 526, 543 (1998).

[25] H. Kleinert and F. S. Nogueira, Phys. Rev. B 66, 012504 (2002).

[26] A. C. Petkou, Ann. Phys. (N.Y.) 249, 180 (1996).

[27] For the conformally invariant $O(n)$ model the current is given by $j_{\mu}^{a b}=\phi^{a} \partial_{\mu} \phi^{b}-\phi^{b} \partial_{\mu} \phi^{a}$ and $\left\langle j_{\mu}^{r s}(x) j_{\nu}^{t u}(0)\right\rangle=$ $k S_{d}^{-2} x^{2(1-d)}\left(\delta^{r t} \delta^{s u}-\delta^{r u} \delta^{s t}\right)$, with the latin indices running from 1 to $n$.

[28] R. K. Kaul and S. Sachdev, Phys. Rev. B 77, 155105 (2008).

[29] A. B. Kuklov, N. V. Prokof'ev, B. V. Svistunov, and M. Troyer, Ann. Phys. (N.Y.) 321, 1602 (2006); S. Kragset, E. Smørgrav, J. Hove, F. S. Nogueira, and A. Sudbø, Phys. Rev. Lett. 97, 247201 (2006).

[30] R. Folk and Y. Holovatch, J. Phys. A 29, 3409 (1996).

[31] A. M. Polyakov, Nucl. Phys. B 120, 429 (1977).

[32] M. E. Peskin, Phys. Lett. B 94, 161 (1980).

[33] M. Lüscher, Nucl. Phys. B 180, 317 (1981).

[34] The value of $k^{\prime}$ in this case was computed for large $n$ by A. V. Chubukov, S. Sachdev, and J. Ye, Phys. Rev. B 49, 11919 (1994).

[35] I. R. Klebanov and A. M. Polyakov, Phys. Lett. B 550, 213 (2002).

[36] A. C. Petkou, JHEP 03, 049 (2003).

[37] A. Ritz and J. Ward, arXiv:0811.4195. 